\title{
A Tabu Search Algorithm for Fast Restoration of Large Area Breakdown in Distribution Systems
}

\author{
Jian LIU, Hongli CHENG, Xiaojun SHI, Jingqiu XU \\ School of Electrical Engineering, Xi'an University of Science and Technology, Xi'an, China \\ Email:edliu@bylink.com.cn,Chhl@xust.edu.cn
}

\begin{abstract}
To restore the distribution systems in emergency states with the minimum load shedding, a novel Tabu search approach is put forward. The set of tripped switches is used as candidate solution. Some virtual tripped nodes are defined at the ends of the terminal nodes and by the source nodes. The neighborhood searching is committed by moving a tripped switch to the adjacent node of its upper stream and down stream, respectively. A Tabu list is formed for the tripped switches. The index is to energize as much as possible loads with as less as possible operated times. The electrical limitations and the voltage criterions are used as constrictions. The global aspiration criterion is adopted. An example is given, which shows that the proposed approach is feasible and can deal with complicated indexes.
\end{abstract}

Keywords: distribution systems, restoration, large area break down, load shedding, Tabu search

\section{Introduction}

Although many achievements have been made on fault isolation and restoration, most of them are for fault on a certain feeder section [1-5].

More serious faults, such as failure on a HV transmission line, a main transformer or a bus, may also occur and sometimes cause large area break down in the distribution systems.

Moreover, in some contingent situations of main transformer over loaded, bulk loads need to be transferred to the adjacent substations and sometimes load shedding is necessary, which is quit similar to the restoration process of large area break down.

A fast restoration approach of large area break down based on numerical optimization is put forward in [6]. Although it is smart, it can only deal with the simple index of the minimum load shedding.

In the practice, some other considerations are also needed to be included, such as the times of switching operation, losses, etc.

Tabu search is a promising evolutionary algorithm, which can deal with complicated indexes and constraints. It has been successfully applied into the distribution network reconfiguration [7].

But the conventional Tabu search based network reconfiguration approaches are not suitable to solve the problem of large area break down restoration with a few load shedding, because the numbers of tripped switches before and after reconfiguration must be equal.

In this paper, a novel Tabu search based approach is proposed to solve above problems.

\section{Basic Principles}

\subsection{Construction of Solutions}

In the Tabu search based distribution network reconfiguration, the set of tripped switches can be used as candidate solution.

The initial solution $\boldsymbol{s}^{(0)}$ based on the current topology of the distribution network is

$$
\boldsymbol{s}^{(0)}=\left[s_{1}^{(0)}, s_{2}^{(0)}, \ldots, s_{M}^{(0)}\right]
$$

where, $s_{i}^{(0)}$ is the sequence number of the $i$-th tripped switch of the current operation mode, $M$ is the number of the tripped switches.

A candidate solution of the $k$-th iteration $\boldsymbol{s}^{(k)}$ is

$$
\boldsymbol{s}^{(k)}=\left[s_{1}^{(k)}, s_{2}^{(k)}, \ldots, s_{N}^{(k)}\right]
$$

If some loads are shed, we have

$$
N>M
$$

\subsection{Virtual Tripped Nodes}

In order to meet the requirement of $N>M$, we should define virtual tripped switches, which are shown in Figure 1.

The initial positions of virtual tripped switches are at the end of terminal nodes and behind the source nodes. The total number of virtual tripped switches is the summation of the number of terminal nodes and the number of the source nodes. 


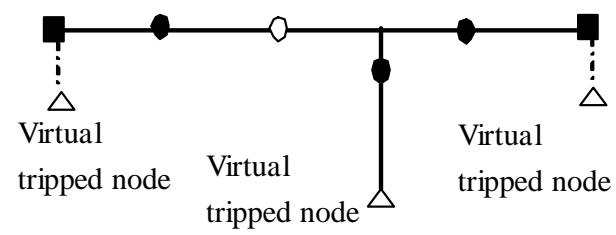

Figure 1. Illustration of virtual tripped switch-nodes

Actual tripped switches and virtual tripped switches are all called tripped switches. With the help of the virtual tripped switches, the Tabu search based distribution network reconfiguration approach can deal with the network reconfiguration problem with load shedding.

In the initial positions, the virtual tripped switches have no influence on the feeder as shown in Figure 1. But when their positions are shifted, some loads may be shed, which are shown in Figures 2(a), (d), (e) and (f). In Figure 2, the reenergized feeder sections are labeled with dotted lines.

\subsection{Neighborhood Searching}

In the iteration of Tabu search, the neighborhood searching is committed, in which, each tripped switch is moved to the adjacent node of its upper stream or down stream

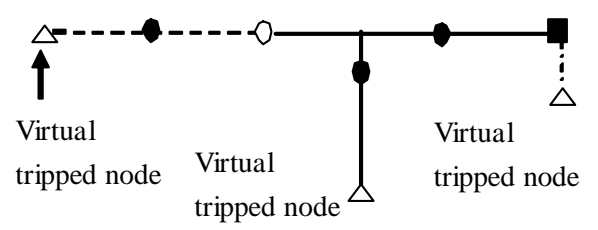

(a)

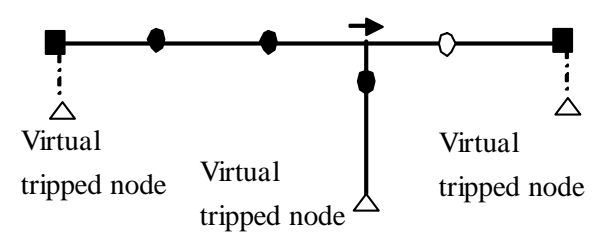

(c)

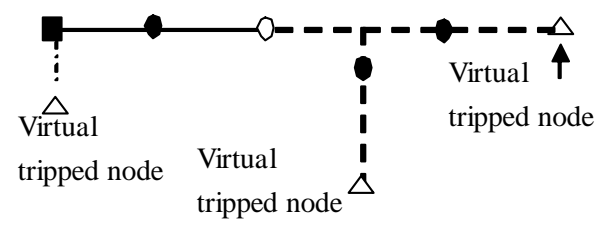

(e) while the other switches keep their states not changed. Therefore, the candidate solution set is formed.

Assuming that the selected solution of the $k$-th iteration is shown in Figure 1, its candidate solutions of the $k+1$-th iteration produced by the neighborhood searching are shown in Figure 2.

The arrows in Figure 2 indicate the shifting direction of a certain tripped switch.

In each iteration, the best solution in the candidate solution set without being tabooed is taken as $\boldsymbol{s}^{(k+1)}$ and the corresponding newly tripped switch is taken into the Tabu list.

The global aspiration criterion is adopted, i.e., once the best candidate in the candidate solution set is superior than the 'best so far' solution, it is taken as $\boldsymbol{s}^{(k+1)}$ and the new 'best so far' solution, no matter whether it is in tabooed state.

Above process is committed repeatedly until the stop criterion of no more improvement or reaching the maximum iteration times is satisfied.

\subsection{Fitness Function}

Complicated fitness functions, i.e., indexes, can be used. As an example, a typical fitness function is

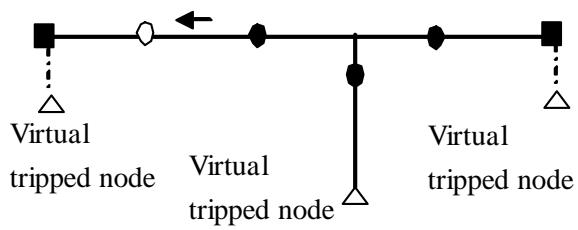

(b)

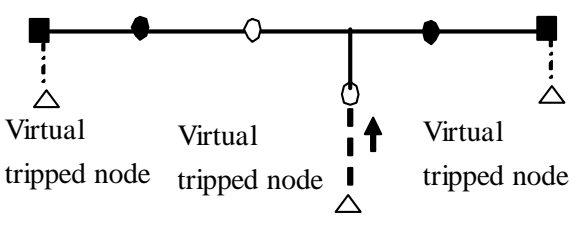

(d)

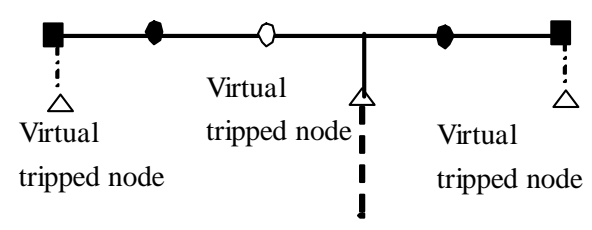

(f)

Figure 2. The candidate solutions 


$$
\operatorname{Max} \quad f=\frac{\beta_{1} \frac{\sum_{i \in \gamma} p_{i}}{P_{\Sigma}}-\beta_{2} \frac{\Delta P}{\sum_{i \in \gamma} p_{i}}}{1+\beta_{3} T}
$$

where, $P_{\Sigma}$ is the total load within the investigating area, $\gamma$ is the set of energized sections after reconfiguration, $\sum_{i \in \gamma} p_{i}$ is the summation of the energized loads after reconfiguration, $\Delta P$ is the total line losses, $T$ is the times of switching operation, $\beta_{1}, \beta_{2}$ and $\beta_{3}$ are weighted values.

The index of (4) indicates that it is encouraged to energize as much as possible loads with as less as possible operating times and line losses.

In most emergency states, the main task is to restore loads as much as possible without considering the line losses. But the difference of importance of various loads should be described in the index. Thus, the fitness function becomes

$$
\operatorname{Max} f=\frac{\sum_{i \in \gamma} k_{i} p_{i}}{1+\beta_{3} T}
$$

where, $k_{i}$ is the weight of the importance of the $i$-th load.

\subsection{Constraints}

Typical constraints are as follows:

-Topology constraints, i.e., no loop exists.

-The times of switching operation should not exceed the maximum value allowed.

-Constraints of electrical limitations, i.e., $I_{i} \leq I_{i, \max }$ where, $I_{i, \max }$ is the current limitation of the $i$-th branch.

-Voltage constraints, i.e., each node voltage is within the range of voltage criterions.

-Other constraints such as locking the switches connected to the malfunctioned buses and keep the switches in reparation in tripped states, etc.

\subsection{Initial Solution}

In contingent situations of main transformer over loaded, the current network topology can be used as the initial solution.

In emergency states due to faults, the steps to form the initial solution are as follows:

Step 1: Isolate of the deenergized buses by tripping all the switches connected to the corresponding buses and lock them in the tripped states, therefore, some feeder sections are deenergized.

Step 2: Close the loop switches (if exist) connecting the deenergized feeder sections to the healthy parts of the distribution network. If there are more than one restora- tion path for a certain deenergized feeder section, choose any one of them.

Step 3: Take the obtained topology as the initial solution.

\subsection{Tabu Length and Stop Criterion}

The ability of escaping from the local optimal points is improved with the Tabu length grows larger. But a larger Tabu length may hamper the convergence. As for a small-scale distribution network, it is possible to face the situation of no feasible candidate solution with a too large Tabu length. Therefore, the selection of Tabu length may be in accordance with the number of nodes of the distribution network. In most cases, it ranges from three to five.

The Tabu search based optimization process will terminate if the best solution remains the same within several successive iterations or the times of iteration reaches its maximum value set before hand.

\subsection{Discussions}

The optimal topology obtained by the above approach may contain redundant switching operations. As for the obtained optimal topology, if a certain switch is in tripped state and its adjacent feeder sections are all deenergized, the tripping operation of the switch is redundant and should be eliminated from the switching schedule.

The following measures may improve the efficiency of the proposed approach:

-Never locate any virtual tripped switch at the end of the feeder sections without sectionalizing switches.

-Only the feeders suffering from the failed apparatus and their corresponding connected parts [8] (In [8], a connected part is defined as a feeder group, in which, the load may be transferred from one feeder to the other) are included in the process of Tabu search.

-The Tabu search based optimization processes are performed in each connected part, respectively.

\section{Case Study}

The approach described in Section 2 will now be illustrated with results for Case Study based on a realistic distribution network shown in Figure 3.

There are two HV transmission line paths and three substations, such as, Sb.A, Sb.B and Sb.C and six main transformers labeled from No.1 Tr. to No.6 Tr. The voltage of primary distribution system is $10 \mathrm{kV}$. There are six $10 \mathrm{kV}$ buses, such as B75, B79, B80, B84, B85 and B89.

The solid circles and the hollow indicate the closed and tripped switches, respectively. The numbers besides the circles are the sequence number of the corresponding nodes.

Assuming that the power factors of the loads are of the same value, the loads can be measured in Ampere. The 


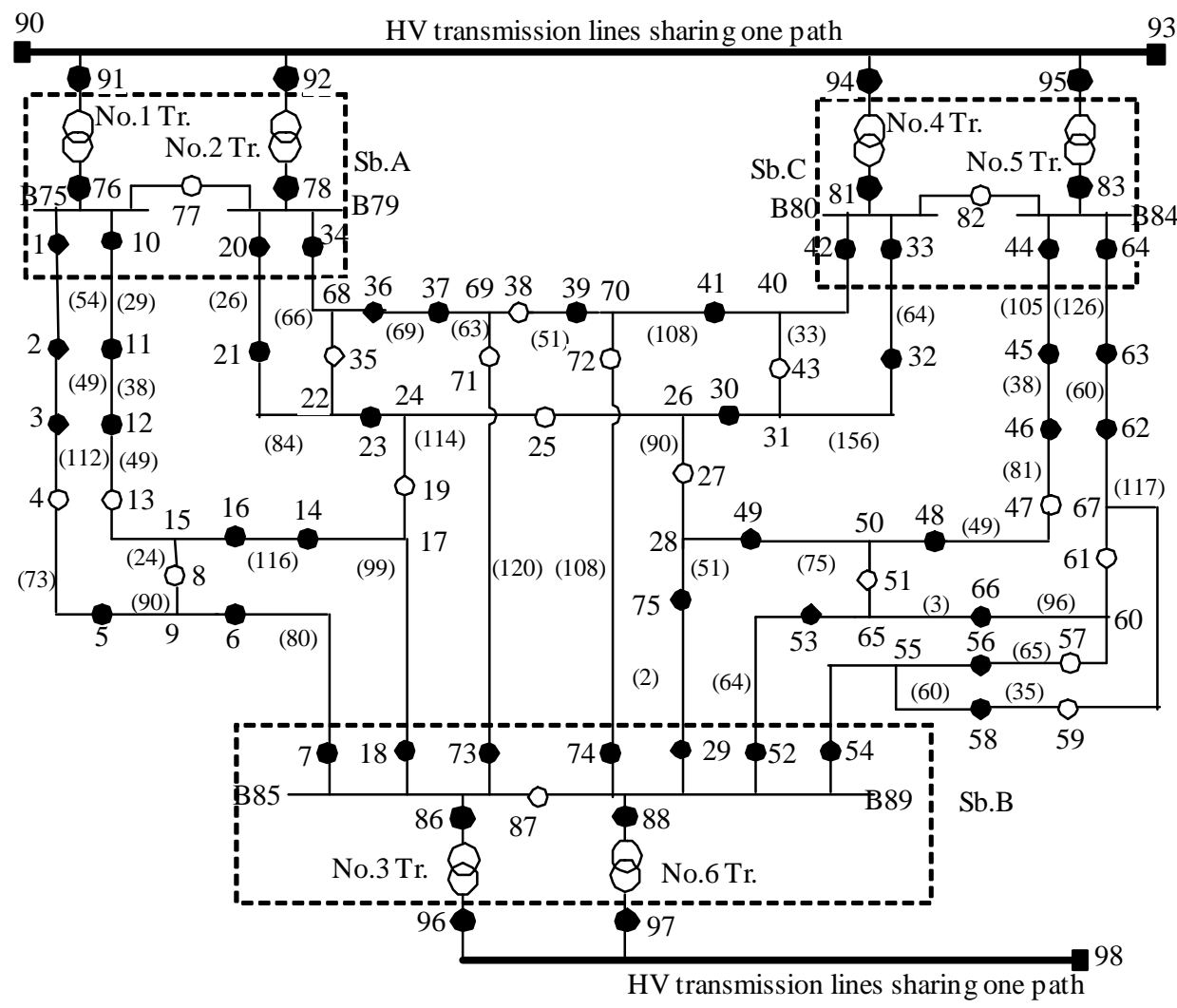

Figure 3. The candidate solutions

numerals in brackets illustrate the amount of loads supplied by the corresponding feeder sections. It can be seen from Figure 3 that the amount of loads is 2992(A) in the normal situation.

The electrical limitation of each main transformer and each bus is $1400(\mathrm{~A})$. The electrical limitation of each feeder is $400(\mathrm{~A})$.

The index is as the form of (5). The Tabu length is set a value of three.

Assuming that B89 fails and both B85 and B89 fail, the restoration schemes produced by the proposed approach are shown in Table 1 and Table 2, respectively.

It can be seen from Table 1 and Table 2 that the times of switching operation may be reduced by increasing the value of $\beta_{3}$ with a little decreasing of the amount of the energized loads, which shows the advantage of the proposed approach than [6].

Table 1. Restoration scheme in case of B89 fails

\begin{tabular}{ccccc}
\hline $\begin{array}{c}\text { Switches tripped } \\
\text { and locked out for } \\
\text { isolation }\end{array}$ & $\beta_{3}$ & $\begin{array}{c}\text { Energized } \\
\text { loads after } \\
\text { restoration }\end{array}$ & $\begin{array}{c}\text { Switching } \\
\text { operation }\end{array}$ & $\begin{array}{c}\text { Switching } \\
\text { times }\end{array}$ \\
\hline & 0.0 & $2768 \mathrm{~A}$ & $\begin{array}{c}\text { switches to trip: } \\
49,53,66 \\
\text { switches to close: } \\
27,47,51,61,72 \\
\text { switches to trip: } \\
69,52,54,74,87\end{array}$ \\
& 0.0015 & 2763A & $\begin{array}{c}66,75 \\
\text { switches to close: } \\
47,61,72\end{array}$ & 5 \\
\hline
\end{tabular}

\section{Conclusions}

1) The way of defining virtual tripped nodes at the end of terminal nodes and by the source nodes is feasible to solve the problem of network reconfiguration with load shedding.

2) Complicated index containing small load shedding, less times of switching operation, lower losses, etc, may be dealt with in the proposed approach.

3) Based on the Distribution Automation System (DAS), the proposed approach is a powerful tool to realize fast restoration of large area break down of distribution systems. It is also useful for bulk loads transferring due to overload of transformers and reparation works.

\section{Acknowledgement}

The authors acknowledge the support of Mr. Zhu Y. Z., Mr. Xue H., Mr. Zhu Y. S. and Mr. Yuan B. for their disinterested support. The authors also esteem the Xi'an Power Supplying Company taking the lead in applying the proposed approach.

\section{REFERENCES}

[1] L. Seung-jae, L. Seng-ii and A. Bok-Shin, “Service res- 
toration of primary distribution systems based on fuzzy evaluation of multi-criteria [J]," IEEE Transaction on Power Systems, Vol. 13, No. 3, pp. 1156-1163. 1998

[2] S. Curcic, C. S. Ozveren, and K. L. Lo, "Computer based strategy for the restoration problem in electric power distribution systems [J]," IEE Proceedings-Generation, Transmission, Distribution, Vol. 144, No. 5, pp. 389-398, 1997.

[3] J. Nahman and G. Strbac, "A new algorithm for service restoration in large-scale urban distribution systems," Electric Power System Research [J], No. 29, pp. 181-192, 1994.

[4] A. Augugliaro, L. Dusonchet, and E. Riva Sansevvrino, "Multi-objective service restoration in distribution networks using an evolutionary approach and fuzzy sets [J],” Electric Power and Energy Systems, No. 22, pp. 103-110, 2000.
[5] A. Pahwa, "Role of distribution automation in restoration of distribution systems after emergencies [C],” Proceedings of the IEEE Power Engineering Society Transmission and Distribution Conference, No. 1, pp. 737-738, 2007.

[6] J. Liu, J. Q. Xu, and H. L. Cheng, "Fast restoration of large area breakdown for power distribution systems [J]," Frontiers of Electrical and Electronic Engineering in China, No. 2, 2006.

[7] G. J. Chen, K. K. Li, and G. Q. Tang, “A Tabu search approach to distribution network reconfiguration for loss reduction [J]," Proceedings of the CSEE, Vol. 22, No. 10, pp. 28-33 (Ch), 2002.

[8] J. Liu, P. X. Bi, and H. P. Dong, "Simplified Analysis and Optimization for Complicated Distribution Systems [M]," China Electric Power Press, Beijing 2002. 